\title{
Privatisierung öffentlicher Dienstleistungen
}

\author{
Hans-Jürgen Bieling \\ Torsten Brandt \\ Thorsten Schulten
}

Seit den frühen 1990er Jahren schreitet die Privatisierung öffentlicher Dienstleistungen in Deutschland voran. Zunächst waren es vor allem die großen nationalen Infrastrukturbereiche wie Post, Telekommunikation, Energie oder die Bahn, die im Mittelpunkt der Privatisierungsbemühungen standen. Mittlerweile hat sich der Fokus der Auseinandersetzungen auf die kommunale Ebene verlagert, wo immer mehr Bereiche der öffentlichen Daseinsvorsorge wie Wohnungen, Entsorgungsunternehmen, Bildungseinrichtungen oder Krankenhäuser zu potenziellen Privatisierungskandidaten werden. Dabei treten neben den vollständigen Verkauf öffentlicher Unternehmen auch immer mehr sogenannte „Public-Private-Partnerships", in denen die Kommunen privaten Unternehmen öffentliche Aufgaben übertragen und ihnen dafür im Gegenzug - oft über einen sehr langfristigen Zeitraum - bestimmte Erträge zusichern.

Hinter der Privatisierung stehen häufig kurzfristige fiskalpolitische Erwägungen, die aus knappen und verschuldeten Haushaltskassen resultieren. Damit geht das große Versprechen einher, dass durch die Privatisierung die Dienstleistungen zukünftig wesentlich effizienter, kostengünstiger und kundenfreundlicher werden, da die Steuerung nicht mehr nach politischen und bürokratischen, sondern nach betriebswirtschaftlichen Kriterien erfolgt. Gesamtwirtschaftlich sollen durch die Privatisierung erhebliche Wohlstandsgewinne erzielt werden, die sich in höheren Wachstums- und Beschäftigungsraten niederschlagen.

Anfangs stieß die Privatisierungswelle nur auf relativ wenig gesellschaftlichen Widerstand. Zu offensichtlich war der Modernisierungsbedarf im öffentlichen Sektor, und es dominierte die Hoffnung, dass es der „Markt" zukünftig besser richten würde. Vor dem Hintergrund von nunmehr fast zwei Jahrzehnten praktischer Privatisierungserfahrungen hat sich das Bild jedoch gedreht. In Meinungsumfragen wird deutlich, dass die Privatisierungspolitik einen erheblichen Legitimationsverlust erlitten hat und dass eine Mehrheit der Bevölkerung sich eine umfassende, durch den Staat abgesicherte, öffentliche Daseinsvorsorge wünscht. Dementsprechend regt sich auch immer mehr Widerstand, was sich beispielsweise an der hohen Anzahl von Bürger- und Volksbegehren ablesen lässt, die sich gegen konkrete Privatisierungsprojekte auf lokaler Ebene wenden. Eine wachsende Zahl von Kommunen ist zudem zu der Erkenntnis gelangt, dass selbst aus einer engen betriebswirtschaftlichen Sicht Privatisierungen sich keineswegs automatisch immer „rechnen“, sondern sich oft als ziemlich teures Unterfangen erweisen. In der Konsequenz lassen sich vermehrt Fälle von Rekommunalisierungen beobachten.

Allerdings ist die Erbringung öffentlicher Dienstleistungen nicht nur eine Frage der Eigentumsform. Mit der Privatisierung eng verbunden ist auch eine Politik der Liberalisierung, durch die alte
Monopolstrukturen aufgebrochen und neue Märkte geschaffen werden. Die „Vermarktlichung“ öffentlicher Dienstleistungen hat zur Folge, dass auch die verbliebenen öffentlichen Unternehmen immer mehr wie ganz normale privatwirtschaftliche Konzerne agieren. Nicht Gemeinwohlorientierung, sondern betriebswirtschaftliche Rationalität bestimmt das Verhalten, und es drohen all diejenigen Bereiche unter die Räder zu kommen, die sich nicht mit dieser Rationalität in Einklang bringen lassen.

Besonders dramatisch sind die Konsequenzen der Liberalisierung und Privatisierung für die Beschäftigten. Insbesondere bei den großen Infrastrukturunternehmen kam es in der Folge der Liberalisierung und Privatisierung zu einem erheblichen Beschäftigungsabbau. Dort, wo im Gegenzug von neu entstandenen Konkurrenz-Unternehmen neue Jobs geschaffen wurden, handelt es sich in vielen Fällen um äußerst prekäre Beschäftigungsverhältnisse. Das Fehlen von Flächentarifverträgen mit branchenweit einheitlichen Tarifstandards bringt es zudem mit sich, dass die neue Konkurrenz in den liberalisierten Dienstleistungsbranchen oft in erster Linie über die Lohnund Arbeitskosten ausgetragen wird. Am Ende stehen nicht mehr Effizienzgewinne, sondern erhebliche soziale Folgekosten.

Die vorliegenden wissenschaftlichen Untersuchungen zeigen mit übergroßer Evidenz, dass die ökonomischen und sozialen Konsequenzen von Privatisierung und Liberalisierung keineswegs die Versprechungen ihrer Befürworter erfüllen. Bis heute weigert sich die Politik jedoch eine nüchterne Bilanz dieser Politik vorzulegen und privatisiert stattdessen munter weiter. Der Deutsche Gewerkschaftsbund fordert deshalb bereits seit Langem von der Bundesregierung, einen Privatisierungsbericht vorzulegen, in dem regelmäßig die ökonomischen und sozialen Folgen analysiert werden. Noch einen Schritt weiter gehen die niederländischen Gewerkschaften, die derzeit für ein Moratorium aller Liberalisierungs- und Privatisierungspolitiken plädieren, das so lange anhalten soll, bis im Rahmen eines breiten gesellschaftlichen Diskurses die Folgen dieser Politik analysiert und entsprechende politische Schlussfolgerungen hieraus gezogen werden.

\footnotetext{
Hans-Jürgen Bieling, Dr., Juniorprofessor am Institut für Politikwissenschaft an der Philipps-Universität Marburg. e-mail: bieling@staff.uni-marburg.de Torsten Brandt, Wissenschaftler im WSI in der Hans-Böckler-Stiftung. e-mail: Torsten-Brandt@boeckler.de

Thorsten Schulten, Dr., Wissenschaftler im WSI in der Hans-BöcklerStiftung. e-mail: Thorsten-Schulten@boeckler.de
} 